\title{
PSEUDO-CHAIN COMPLETENESS OF FORMAL INTERVAL-VALUED FUZZY LOGIC
}

\author{
B. VAN GASSE*, C. CORNELIS, G. DESCHRIJVER and E.E. KERRE \\ Fuzziness and Uncertainty Modelling Research Unit \\ Department of Applied Mathematics and Computer Science \\ Ghent University, Krijgslaan 281 (S9), 9000 Gent, Belgium \\ *E-mail: Bart.VanGasse@UGent.be \\ www.fuzzy. UGent.be
}

\begin{abstract}
Triangle Logic is a formal fuzzy logic with intervals as truth values. Its construction is based on triangle algebras: equationally defined structures that are equivalent with certain residuated lattices on a set of intervals, which were called interval-valued residuated lattices (IVRLs). We prove that the so-called pseudo-prelinear triangle algebras are subdirect products of pseudo-linear triangle algebras. This can be compared with MTL-algebras (prelinear residuated lattices) being subdirect products of linear residuated lattices. Using this result, we prove an analogue of the chain completeness of MTL for Pseudo-prelinear Triangle Logic. It also enables us to prove properties of pseudo-prelinear triangle algebras more easily. We give some examples.
\end{abstract}

Keywords: Interval-valued fuzzy set theory; Residuated lattices; Formal logic.

\section{Introduction}

In order to construct a formal fuzzy logic with (closed) intervals as truth values, the variety of triangle algebras (expansions of residuated lattices) was introduced in Ref. 1 . The idea behind interval-valued truth degrees is that they provide a way to express imprecision as well as gradedness. It was proven that triangle algebras are equivalent with IVRLs, i.e., the residuated lattices in which the exact elements (i.e., the intervals of the form $[a, a]$ ) form a subalgebra. Triangle Logic (TL) was then introduced and proven to be sound and complete w.r.t. triangle algebras.

In this paper, we introduce the concept of filters on triangle algebras and use it to prove that pseudo-prelinear triangle algebras are subdirect products of pseudo-linear triangle algebras. This is analogous to the property that prelinear residuated lattices are subdirect products of linear residu- 
ated lattices. ${ }^{2}$ That result was used to show that MTL is chain complete. In a similar way we obtain that TL is pseudo-chain complete, i.e., complete w.r.t. pseudo-linear triangle algebras.

\section{Preliminaries}

Definition 2.1. A residuated lattice is a structure $\mathcal{L}=(L, \sqcap, \sqcup, *, \Rightarrow, 0,1)$ in which $\sqcap, \sqcup, *$ and $\Rightarrow$ are binary operators on the set $L$ and

- $(L, \sqcap, \sqcup)$ is a bounded lattice with 0 as smallest and 1 as greatest element,

- * is commutative and associative, with 1 as neutral element, and

- $x * y \leq z$ iff $x \leq y \Rightarrow z$ for all $x, y$ and $z$ in $L$ (residuation principle).

An MTL-algebra ${ }^{2}$ is a prelinear residuated lattice, i.e., a residuated lattice in which $(x \Rightarrow y) \sqcup(y \Rightarrow x)=1$ for all $x$ and $y$ in $L$.

A BL-algebra ${ }^{3}$ is a divisible MTL-algebra, i.e., an MTL-algebra in which $x \sqcap y=x *(x \Rightarrow y)$ for all $x$ and $y$ in $L$. The weaker property $x \sqcap y=$ $(x *(x \Rightarrow y)) \sqcup(y *(y \Rightarrow x))$ is called weak divisibility. ${ }^{1,4}$

If a residuated lattice satisfies $x \sqcup y=((x \Rightarrow y) \Rightarrow y) \sqcap((y \Rightarrow x) \Rightarrow x)$, for all $x$ and $y$ in $L$, then it is called $\sqcup$-definable.

We will use the notations $\neg x$ for $x \Rightarrow 0, x \Leftrightarrow y$ for $(x \Rightarrow y) \sqcap(y \Rightarrow x)$.

Definition 2.2. Given a lattice $\mathcal{L}=(L, \sqcap, \sqcup)$, its triangularization $\mathbb{T}(\mathcal{L})$ is the structure $\mathbb{T}(\mathcal{L})=(\operatorname{Int}(\mathcal{L}), \sqcap, \sqcup)$ defined by

- $\operatorname{Int}(\mathcal{L})=\left\{\left[x_{1}, x_{2}\right] \mid\left(x_{1}, x_{2}\right) \in L^{2}\right.$ and $\left.x_{1} \leq x_{2}\right\}$

- $\left[x_{1}, x_{2}\right] \sqcap\left[y_{1}, y_{2}\right]=\left[x_{1} \sqcap y_{1}, x_{2} \sqcap y_{2}\right]$

- $\left[x_{1}, x_{2}\right] \sqcup\left[y_{1}, y_{2}\right]=\left[x_{1} \sqcup y_{1}, x_{2} \sqcup y_{2}\right]$

The set $D_{\mathcal{L}}=\{[x, x] \mid x \in L\}$ is called the diagonal of $\mathbb{T}(\mathcal{L})$. The triangularization of $([0,1]$, min, max $)$ is denoted as $\mathcal{L}^{I}=\left(L^{I}, \sqcap, \sqcup\right)$.

Definition 2.3. An interval-valued residuated lattice (IVRL) is a residuated lattice $\left(\operatorname{Int}(\mathcal{L}), \sqcap, \sqcup, \odot, \Rightarrow_{\odot},[0,0],[1,1]\right)$ on the triangularization $\mathbb{T}(\mathcal{L})$ of a bounded lattice $\mathcal{L}$, in which $D_{\mathcal{L}}$ is closed under $\odot$ and $\Rightarrow_{\odot}$, i.e., $\left[x_{1}, x_{1}\right] \odot\left[y_{1}, y_{1}\right] \in D_{\mathcal{L}}$ and $\left[x_{1}, x_{1}\right] \Rightarrow_{\odot}\left[y_{1}, y_{1}\right] \in D_{\mathcal{L}}$ for all $x_{1}, y_{1}$ in $L$.

In Ref. 1, we introduced the notion of triangle algebra, as an equational representation for an interval-valued residuated lattice. 
Definition 2.4. A triangle algebra is a structure $\mathcal{A}=(A, \sqcap, \sqcup, *, \Rightarrow$, $\nu, \mu, 0, u, 1)$, in which $(A, \sqcap, \sqcup, *, \Rightarrow, 0,1)$ is a residuated lattice, $\nu$ and $\mu$ are binary operators, $u$ a constant, and satisfying the following conditions:

$$
\begin{array}{ll}
T .1 \quad \nu x \leq x, & T .1^{\prime} x \leq \mu x, \\
T .2 \quad \nu x \leq \nu \nu x, & T .2^{\prime} \mu \mu x \leq \mu x, \\
T .3 \quad \nu(x \sqcap y)=\nu x \sqcap \nu y, & T .3^{\prime} \mu(x \sqcap y)=\mu x \sqcap \mu y, \\
T .4 \quad \nu(x \sqcup y)=\nu x \sqcup \nu y, & T .4^{\prime} \mu(x \sqcup y)=\mu x \sqcup \mu y, \\
T .5 \quad \nu 1=1, & T .5^{\prime} \mu 0=0, \\
T .6 \quad \nu u=0, & T .6^{\prime} \mu u=1, \\
T .7 \quad \nu \mu x=\mu x, & T .7^{\prime} \mu \nu x=\nu x, \\
T .8 \quad \nu(x \Rightarrow y) \leq \nu x \Rightarrow \nu y, & \\
T .9 \quad \nu x \Leftrightarrow \nu y) *(\mu x \Leftrightarrow \mu y) \leq(x \Leftrightarrow y), & \\
T .10 \nu x \Rightarrow \nu y \leq \nu(\nu x \Rightarrow \nu y) . &
\end{array}
$$

Definition 2.5. Let $\mathcal{A}=(A, \sqcap, \sqcup, *, \Rightarrow, \nu, \mu, 0, u, 1)$ be a triangle algebra. An element $x$ in $A$ is called exact if $\nu x=x$. The set of exact elements of $\mathcal{A}$ is denoted by $E(\mathcal{A})$.

Every property in Definition 2.1 (prelinearity, divisibility, ...) can be weakened, by imposing it on $E(\mathcal{A})$ (instead of $A$ ) only. We will denote this with the prefix 'pseudo'. For example, a triangle algebra is said to be pseudolinear if its set of exact elements is linearly ordered (by the original (restricted) ordering). Another example: a triangle algebra is pseudo-divisible if $\nu x \sqcap \nu y=\nu x *(\nu x \Rightarrow \nu y)$ for all $x$ and $y$ in $A$ (because $E(\mathcal{A})$ consists exactly of the elements of the form $\nu x)$.

In Ref. 1, we established a one-to-one correspondence between IVRLs and triangle algebras. The unary operators $\nu$ and $\mu$ correspond with the mappings that map $\left[x_{1}, x_{2}\right]$ to $\left[x_{1}, x_{1}\right]$ and $\left[x_{2}, x_{2}\right]$ respectively. The constant $u$ corresponds to $[0,1]$.

In the same article, we introduced Triangle Logic (TL). It is basically Monoidal Logic ${ }^{5}$ (ML) enriched with three connectives: $\perp, \square$ and $\diamond$ (corresponding with $u, \nu$ and $\mu$, respectively). The axioms are those of ML, extended with the 'translations' of T.1-T.10 and T.1'-T.7'. The deduction rules are modus ponens (MP, from $\phi$ and $\phi \rightarrow \psi$ infer $\psi$ ), generalization (G, from $\phi$ infer $\square \phi)$ and monotonicity of $\diamond(\mathrm{M} \diamond$, from $\phi \rightarrow \psi$ infer $\diamond \phi \rightarrow \diamond \psi)$. Theories, provability, evaluations and models are defined in the usual way. $\mathrm{TL}$ is sound and complete w.r.t. triangle algebras, and axiomatic extensions 
of this expansion of ML are sound and complete w.r.t. the corresponding subclasses of the class of triangle algebras.

In Ref. 6 , we proved that in a triangle algebra $\mathcal{A}, *$ and $\Rightarrow$ are determined by their action on $E(\mathcal{A})$ and the value of $u * u$. This result implies that the only IVRLs on $L^{I}$ are those that are considered in Ref. 4, based on a t-norm of the form $\mathcal{T}_{T, \alpha}\left(\left[x_{1}, x_{2}\right],\left[y_{1}, y_{2}\right]\right)=$ $\left[T\left(x_{1}, y_{1}\right), \max \left(T\left(\alpha, T\left(x_{2}, y_{2}\right)\right), T\left(x_{1}, y_{2}\right), T\left(x_{2}, y_{1}\right)\right)\right]$, with $\alpha \in[0,1]$.

\section{Filters and completeness results}

Definition 3.1. Let $\mathcal{A}=(A, \sqcap, \sqcup, *, \Rightarrow, \nu, \mu, 0, u, 1)$ be a triangle algebra. A filter of $\mathcal{A}$ is a non-empty subset $F$ of $A$, satisfying:

(1) if $x \in F, y \in A$ and $x \leq y$, then $y \in F$;

(2) if $x \in F$, then $\nu x \in F$;

(3) if $x, y \in F$, then $x * y \in F$.

A filter $F$ of $\mathcal{A}$ is called a prime filter of $\mathcal{A}$ iff for every $x$ and $y$ in $A$, at least one element of $\{\nu x \Rightarrow \nu y, \nu y \Rightarrow \nu x\}$ is in $F$. For all $x$ and $y$ in $A$, we write $x \sim_{F} y$ iff $x \Rightarrow y$ and $y \Rightarrow x$ are both in $F$.

Proposition 3.1. Let $F$ be a filter in a triangle algebra $\mathcal{A}=(A, \sqcap, \sqcup, *$, $\Rightarrow, \nu, \mu, 0, u, 1)$. Then the relation $\sim_{F}$ is a congruence relation on $\mathcal{A}$.

As a corollary, we can meaningfully consider the quotient algebra $\mathcal{A}_{F}=$ $\left(A_{F}, \sqcap_{F}, \sqcup_{F}, *_{F}, \Rightarrow_{F}, \nu_{F}, \mu_{F},[0]_{F},[u]_{F},[1]_{F}\right)$, in which $A_{F}$ is the set of equivalence classes (induced by $\sim_{F}$ ) in $A$, and for any $x$ and $y$ in $A$, $[x]_{F}=\left\{y \in A \mid x \sim_{F} y\right\},[x]_{F} \odot_{F}[y]_{F}=[x \odot y]_{F}($ for $\odot \in\{\sqcap, \sqcup, *, \Rightarrow\})$, $\nu_{F}[x]_{F}=[\nu x]_{F}$ and $\mu_{F}[x]_{F}=[\mu x]_{F}$. Then $\mathcal{A}_{F}$ is a triangle algebra.

Proposition 3.2. Let $\mathcal{A}=(A, \sqcap, \sqcup, *, \Rightarrow, \nu, \mu, 0, u, 1)$ be a triangle algebra and $F$ a filter in $\mathcal{A}$. Then $F$ is a prime filter iff $E\left(\mathcal{A}_{F}\right)$ is linearly ordered.

Proposition 3.3. Let $\mathcal{A}$ be a pseudo-prelinear triangle algebra and $a \in$ $A \backslash\{1\}$. Then there exists a prime filter $F$ in A not containing $a$.

Exactly as in Ref. 3, we can use the previous two propositions to prove

Proposition 3.4. Every pseudo-prelinear triangle algebra $\mathcal{A}$ is a subalgebra of the direct product of a system of pseudo-linear triangle algebras.

This proposition is also valid for subvarieties of pseudo-prelinear triangle algebras. For example, every $\sqcup$-definable pseudo-prelinear triangle algebra 
is a subalgebra of the direct product of a system of $\sqcup$-definable pseudo-linear triangle algebras.

Definition 3.2. Pseudo-linear Triangle Logic (PTL) is the axiomatic extension of TL, obtained by adding axiom scheme $(\square \phi \rightarrow \square \psi) \vee(\square \psi \rightarrow \square \phi)$.

Together with the completeness result in Ref. 1 (which is the equivalence of the first two statements in Theorem 3.1), Proposition 3.4 implies:

Theorem 3.1. For each formula $\phi$, the following are equivalent:

- $\phi$ can be deduced from a theory $V$ in PTL,

- for every pseudo-prelinear triangle algebra $\mathcal{A}$ and for every $\mathcal{A}$-model $e$ of $V, e(\phi)=1$,

- for every pseudo-linear triangle algebra $\mathcal{A}$ and for every $\mathcal{A}$-model e of $V, e(\phi)=1$.

This completeness results remains valid for schematic extensions of PTL. Some examples of axioms that could be added are discussed in Ref. 1.

Another consequence of Proposition 3.4 is that identities valid in every pseudo-linear triangle algebra remain valid in every pseudo-prelinear triangle algebra. In Ref. 4, we proved a number of properties for a class of t-norms on $\mathcal{L}^{I}$. In the proofs, no specific t-norms are considered and apart from the linearity of the diagonal - no properties of $\mathcal{L}^{I}$ are used. So - using the one-to-one correspondence between (pseudo-linear) IVRLs and (pseudo-linear) triangle algebras - we can conclude that these properties are even valid in every pseudo-linear triangle algebra. Many of these properties (e.g. all identities) are therefore valid in every pseudo-prelinear triangle algebra. We give some examples:

(1) Distributivity. One can easily prove that this holds in every pseudolinear triangle algebra. As distributivity is expressed with an identity, we can immediately state that it also holds in every pseudo-prelinear triangle algebra.

(2) Weak divisibility is equivalent with pseudo-divisibility, i.e. every pseudo-prelinear triangle algebra satisfying $x \sqcap y=(x *(x \Rightarrow y)) \sqcup$ $(y *(y \Rightarrow x))$ also satisfies $\nu x \sqcap \nu y=\nu x *(\nu x \Rightarrow \nu y)$, and vice versa. Indeed, every weak divisible pseudo-prelinear triangle algebra is a subdirect product of weak divisible pseudo-linear triangle algebras (which are known to be pseudo-divisible ${ }^{4}$. As a subdirect product of pseudo- 
divisible triangle algebras, it is pseudo-divisible. The argument for the converse (and the examples to come) is completely analogous.

(3) Every $\sqcup$-definable pseudo-prelinear triangle algebra that satisfies $u * u=$ $u$ (or equivalently $\mu(u * u)=1$ ), also satisfies $\nu x \sqcup \nu y=(\nu x \Rightarrow \nu y) \Rightarrow \nu y$.

(4) Every pseudo-prelinear triangle algebra satisfying $\nu x \sqcup \nu y=(\nu x \Rightarrow$ $\nu y) \Rightarrow \nu y$, is $\sqcup$-definable.

\section{Conclusion and Future Work}

We defined filters on triangle algebras and used them to demonstrate that pseudo-prelinear triangle algebras are subdirect products of pseudo-linear triangle algebras. As a consequence, some properties hold for all pseudoprelinear triangle algebras if they hold for all pseudo-linear triangle algebras. We gave some examples of such properties. Another corollary is that the completeness theorem for Pseudo-prelinear Triangle Logic can be strenghtened. Maybe this completeness theorem can be reinforced even more, to standard completeness (i.e., w.r.t. the triangle algebras on $\mathcal{L}^{I}$ ). This is a topic for further research.

\section{Acknowledgments}

Bart Van Gasse and Chris Cornelis would like to thank the Research Foundation-Flanders for funding their research.

\section{References}

1. B. Van Gasse, C. Cornelis, G. Deschrijver and E.E. Kerre, Triangle algebras: A formal logic approach to interval-valued residuated lattices, Fuzzy Sets and Systems, in press.

2. F. Esteva and L. Godo, Monoidal t-norm based logic: Towards a logic for left-continuous t-norms, Fuzzy Sets and Systems 124, 271-288 (2001).

3. P. Hájek, Metamathematics of Fuzzy Logic (Kluwer Academic Publishers, 1998).

4. B. Van Gasse, C. Cornelis, G. Deschrijver and E.E. Kerre, On the properties of a generalized class of t-norms in interval-valued fuzzy logics, New Mathematics and Natural Computation 2, 29-42 (2006).

5. U. Höhle, Commutative, Residuated l-monoids, in Non-classical Logics and their Applications to Fuzzy Subsets: a Handbook of the Mathematical Foundations of Fuzzy Set Theory, eds. U. Höhle and E.P. Klement (Kluwer Academic Publishers, 1995), pp. 53-106.

6. B. Van Gasse, C. Cornelis, G. Deschrijver and E.E. Kerre, A characterization of interval-valued residuated lattices, submitted. 